\title{
Lamb Shift for static atoms outside a Schwarzschild black hole
}

\author{
Wenting Zhou ${ }^{1}$ and Hongwei $\mathrm{Yu}^{1,2 *}$ \\ 1 Department of Physics and Key Laboratory of Low Dimensional \\ Quantum Structures and Quantum Control of Ministry of Education, \\ Hunan Normal University, Changsha, Hunan 410081, China \\ 2 Center for Nonlinear Science and Department of Physics, \\ Ningbo University, Ningbo, Zhejiang 315211, China
}

\begin{abstract}
We study, by separately calculating the contributions of vacuum fluctuations and radiation reaction to the atomic energy level shift, the Lamb shift of a static two-level atom interacting with real massless scalar fields in the Boulware, Unruh and Hartle-Hawking vacuums outside a Schwarzschild black hole. We find that in the Boulware vacuum, the Lamb shift gets a correction arising as a result of the backscattering of vacuum field modes off the space-time curvature, which is reminiscent of the correction to the Lamb shift induced by the presence of cavities. However, when the Unruh and Hartle-Hawking vacua are concerned, our results show that the Lamb shift behaves as if the atom were irradiated by a thermal radiation or immersed in a thermal bath at the Hawking temperature, depending on whether the scalar field is in the Unruh or the Hartle-Hawking vacuum. Remarkably, the thermal radiation is always backscattered by the space-time geometry.

PACS numbers: 04.70.Dy, 04.62.+v, 12.20.Ds, 97.60.Lf
\end{abstract}

\footnotetext{
* Corresponding author
} 


\section{INTRODUCTION}

The Lamb shift, the shift of the energy levels of an atom that arises as a result of the coupling of the atom to vacuum fluctuations of quantum fields in a flat space-time, is one of the most remarkable observable effects predicted by quantum field theory. Later, it has been shown that this radiative energy level shift of an atom can be modified by the presence of cavities [1] and the noninertial motion of the atom itself [2 5], which alter the vacuum fluctuations and yield a Lamb shift different from its original value.

Here, we are concerned with the Lamb shift of a static atom in the exterior of a spherically symmetric black hole interacting with vacuum fluctuations of quantum fields. When a curved space-time is considered as opposed to a flat one, a delicate issue then arises as to how the vacuum state of the quantum fields is determined. Normally, a vacuum state is associated with nonoccupation of positive frequency modes. However, the positive frequency of field modes is defined with respect to the time coordinate. Therefore, to define positive frequency, one has to first specify a definition of time. In a spherically symmetric black hole background, one definition is the Schwarzschild time, $t$, and it is a natural definition of time in the exterior region. The vacuum state, defined by requiring normal modes to be positive frequency with respect to the Killing vector $\partial / \partial t$ with respect to which the exterior region is static, is called the Boulware vacuum. Other possibilities that have been proposed are the Unruh vacuum [6] and the Hartle-Hawking vacuum [7]. The Unruh vacuum is defined by taking modes that are incoming from $\mathscr{J}^{-}$to be positive frequency with respect to $\partial / \partial t$, while those that emanate from the past horizon are taken to be positive frequency with respect to the Kruskal coordinate $\bar{u}$, the canonical affine parameter on the past horizon. The Hartle-Hawking vacuum, on the other hand, is defined by taking the incoming modes to be positive frequency with respect to $\bar{v}$, the canonical affine parameter on the future horizon, and outgoing modes to be positive frequency with respect to $\bar{u}$. The calculations of the values of physical observables, such as the expectation values of the energy-momentum tensor and the response rate of an Unruh detector in these vacuum states, have yielded the following physical understanding: (i) The Boulware vacuum corresponds to our familiar concept of a vacuum state at large radii. It would be the vacuum state outside a massive spherical body of radius only slight larger than its Schwarzschild radius; (ii) the Unruh vacuum is the vacuum state that best approximates the state that we would obtain following the gravitational collapse of a massive body to a black hole; (iii) the Hartle-Hawking state, however, does not correspond to our usual notion of a vacuum, but describes a black hole in equilibrium with a sea of thermal radiation.

In the current paper, we will study the Lamb shift of a static two-level atom outside a fourdimensional Schwarzschild black hole in interaction with massless quantum scalar fields in all the above three vacuum states. It should be pointed out that a fully realistic treatment of the Lamb shift requires considering a multilevel atom coupled to the electromagnetic field. However, the purpose of the present paper is to bring to light the essential features of the full problem in a simple model and keep the discussion as clear and transparent as 
possible. Our calculations of the Lamb shift make use of an elegant formalism suggested by Dalibard, Dupont-Roc, and Cohen-Tannoudji(DDC) [8, 9] which allows a separation of the contributions of vacuum fluctuations and the radiation reaction to the energy shifts. This separation is also interesting from a conceptual point of view since the Lamb shift is usually associated with vacuum fluctuations alone. In previous studies [10], we have separately calculated the contributions of vacuum fluctuations and the radiation reaction to the spontaneous excitation rate of a static atom outside a Schwarzschild black hole, and find that the atom would spontaneously excites as if it were irradiated by or immersed in a thermal radiation at the Hawking temperature.

\section{GENERAL FORMALISM}

Let us consider a two-level atom in interaction with a quantum real massless scalar field outside a Schwarzschild black hole. The metric of the space-time can be written in terms of the Schwarzschild coordinates as

$$
d s^{2}=\left(1-\frac{2 M}{r}\right) d t^{2}-\left(1-\frac{2 M}{r}\right)^{-1} d r^{2}-r^{2}\left(d \theta^{2}+\sin ^{2} \theta d \varphi^{2}\right),
$$

where $M$ is the mass of the black hole. Without loss of generality, we assume a pointlike two-level atom on a stationary space-time trajectory $x(\tau)$, where $\tau$ denotes the proper time on the trajectory. The stationarity of the trajectory guarantees the existence of stationary atomic states, $|+\rangle$ and $|-\rangle$, with energies $\pm \frac{1}{2} \omega_{0}$ and a level spacing $\omega_{0}$. The Hamiltonian that describes the time evolution of the atom-field interacting system can be given by [2, 11]

$$
H(\tau)=H_{A}(\tau)+H_{F}(\tau)+H_{I}(\tau)
$$

where

$$
\begin{gathered}
H_{A}(\tau)=\hbar \omega_{0} S_{z}(\tau) \\
H_{F}(\tau)=\int d^{3} k \hbar \omega_{\vec{k}} a_{\vec{k}}^{\dagger} a_{\vec{k}} \frac{d t}{d \tau} \\
H_{I}(\tau)=\mu S_{2}(\tau) \phi(x(\tau))
\end{gathered}
$$

Here $a_{\vec{k}}^{\dagger}$ and $a_{\vec{k}}$ are the creation and annihilation operators for a scalar particle with momentum $\vec{k}$, and $\mu$ is a coupling constant which we assume to be small. $S_{z}, S_{+}$, and $S_{-}$are the pseudospin operators of the atom, $S_{z}(0)=(1 / 2)(|+\rangle\langle+|-|-\rangle\langle-|)$ and $S_{2}(0)=(i / 2)\left(S_{-}-S_{+}\right)$. Here $S_{+}(0)=|+\rangle\langle-|$, and $S_{-}(0)=|-\rangle\langle+|$. The field operators $\phi$ is evaluated along the trajectory $x(\tau)$ of the atom.

Then we can write down the Heisenberg equations of motion for the dynamical variables of the atom and field from the Hamiltonian of the system in Eq. (2). The solutions of the equations of motion can be split into the two parts: a free part, which is present even in the absence of the coupling, and a source part, which is caused by the interaction of the 
atom and field. We assume that the initial state of the atom is $|b\rangle$ and the scalar field is in a vacuum state $|0\rangle$. To identify the two distinct contributions of vacuum fluctuations and radiation reaction to the energy level shift of a two-level atom, we use DDC's formalism to choose a symmetric ordering between the atom and field variables, and separate the vacuum fluctuations and radiation reaction contributions to the rate of change of an arbitrary atomic observable $G(\tau)$. Following the procedures that have been shown in Refs. [2, 9], we take the average over the vacuum state of the scalar field, and obtain

$$
\left\langle 0\left|\left(\frac{d G(\tau)}{d \tau}\right)_{v f, r r}\right| 0\right\rangle=\frac{i}{\hbar}\left[H_{v f, r r}^{e f f}(\tau), G(\tau)\right]+\text { non-Hamiltonian terms },
$$

where the non-Hamiltonian parts are related to relaxation effects, and in order $\mu^{2}$, the effective Hamiltonians can be expressed as

$$
\begin{aligned}
& H_{v f}^{e f f}(\tau)=\frac{i \mu^{2}}{2 \hbar} \int_{\tau_{0}}^{\tau} d \tau^{\prime} C^{F}\left(x(\tau), x\left(\tau^{\prime}\right)\right)\left[S_{2}^{f}(\tau), S_{2}^{f}\left(\tau^{\prime}\right)\right] \\
& H_{r r}^{e f f}(\tau)=-\frac{i \mu^{2}}{2 \hbar} \int_{\tau_{0}}^{\tau} d \tau^{\prime} \chi^{F}\left(x(\tau), x\left(\tau^{\prime}\right)\right)\left\{S_{2}^{f}(\tau), S_{2}^{f}\left(\tau^{\prime}\right)\right\}
\end{aligned}
$$

Here [, ] and $\{$,$\} are the commutator and anti-commutator respectively, and the subscripts$ " $v f$ " stands for vacuum fluctuations and " $r$ " for radiation reaction. The statistical functions of the field $C^{F}$ and $\chi^{F}$ are also called symmetric correlation function and linear susceptibility of the field. They are defined as

$$
\begin{aligned}
C^{F}\left(x(\tau), x\left(\tau^{\prime}\right)\right) & =\frac{1}{2}\left\langle 0\left|\left\{\phi^{f}(x(\tau)), \phi^{f}\left(x\left(\tau^{\prime}\right)\right)\right\}\right| 0\right\rangle, \\
\chi^{F}\left(x(\tau), x\left(\tau^{\prime}\right)\right) & =\frac{1}{2}\left\langle 0\left|\left[\phi^{f}(x(\tau)), \phi^{f}\left(x\left(\tau^{\prime}\right)\right)\right]\right| 0\right\rangle .
\end{aligned}
$$

Taking the expectation value of Eqs. (77) and (8) in the atom's initial state $|b\rangle$, we can obtain the radiative energy shifts of the atom's level $|b\rangle$ due to the vacuum fluctuations and radiation reaction,

$$
\begin{aligned}
& \left(\delta E_{b}\right)_{v f}=-\frac{i \mu^{2}}{\hbar} \int_{\tau_{0}}^{\tau} d \tau^{\prime} C^{F}\left(x(\tau), x\left(\tau^{\prime}\right)\right)\left(\chi^{A}\right)_{b}\left(\tau, \tau^{\prime}\right) \\
& \left(\delta E_{b}\right)_{r r}=-\frac{i \mu^{2}}{\hbar} \int_{\tau_{0}}^{\tau} d \tau^{\prime} \chi^{F}\left(x(\tau), x\left(\tau^{\prime}\right)\right)\left(C^{A}\right)_{b}\left(\tau, \tau^{\prime}\right)
\end{aligned}
$$

where $\left(C^{A}\right)_{b}$ and $\left(\chi^{A}\right)_{b}$, the symmetric correlation function and the linear susceptibility of the atom, are defined as

$$
\begin{aligned}
\left(C^{A}\right)_{b}\left(\tau, \tau^{\prime}\right) & =\frac{1}{2}\left\langle b\left|\left\{S_{2}^{f}(\tau), S_{2}^{f}\left(\tau^{\prime}\right)\right\}\right| b\right\rangle, \\
\left(\chi^{A}\right)_{b}\left(\tau, \tau^{\prime}\right) & =\frac{1}{2}\left\langle b\left|\left[S_{2}^{f}(\tau), S_{2}^{f}\left(\tau^{\prime}\right)\right]\right| b\right\rangle
\end{aligned}
$$


which are characterized by the atom itself. Explicitly, the statistical functions of the atom can be given as

$$
\begin{aligned}
\left(C^{A}\right)_{b}\left(\tau, \tau^{\prime}\right) & =\frac{1}{2} \sum_{d}\left|\left\langle b\left|S_{2}(0)\right| d\right\rangle\right|^{2}\left(e^{i \omega_{b d} \Delta \tau}+e^{-i \omega_{b d} \Delta \tau}\right), \\
\left(\chi^{A}\right)_{b}\left(\tau, \tau^{\prime}\right) & =\frac{1}{2} \sum_{d}\left|\left\langle b\left|S_{2}(0)\right| d\right\rangle\right|^{2}\left(e^{i \omega_{b d} \Delta \tau}-e^{-i \omega_{b d} \Delta \tau}\right),
\end{aligned}
$$

where $\omega_{b d}=\omega_{b}-\omega_{d}, \Delta \tau=\tau-\tau^{\prime}$ and the sum extends over a complete set of atomic states.

\section{LAMB SHIFTS OF STATIC ATOMS OUTSIDE A BLACK HOLE.}

In the exterior region of the Schwarzschild black hole, a complete set of normalized basis functions for the massless scalar field that satisfy the Klein-Gordon equation is given by

$$
\begin{aligned}
& \vec{u}_{\omega l m}=(4 \pi \omega)^{-\frac{1}{2}} e^{-i \omega t} \vec{R}_{l}(\omega \mid r) Y_{l m}(\theta, \varphi) \\
& \overleftarrow{u}_{\omega l m}=(4 \pi \omega)^{-\frac{1}{2}} e^{-i \omega t} \overleftarrow{R}_{l}(\omega \mid r) Y_{l m}(\theta, \varphi)
\end{aligned}
$$

where $Y_{l m}(\theta, \varphi)$ are the spherical harmonics and the radial functions have the following asymptotic forms [12]

$$
\begin{aligned}
& \vec{R}_{l}(\omega \mid r) \sim \begin{cases}r^{-1} e^{i \omega r_{*}}+\vec{A}_{l}(\omega) r^{-1} e^{-i \omega r_{*}}, & r \rightarrow 2 M, \\
B_{l}(\omega) r^{-1} e^{i \omega r_{*}}, & r \rightarrow \infty,\end{cases} \\
& \overleftarrow{R}_{l}(\omega \mid r) \sim \begin{cases}B_{l}(\omega) r^{-1} e^{-i \omega r_{*}}, & r \rightarrow 2 M, \\
r^{-1} e^{-i \omega r_{*}}+\overleftarrow{A}_{l}(\omega) r^{-1} e^{i \omega r_{*}}, & r \rightarrow \infty,\end{cases}
\end{aligned}
$$

with $r_{*}=r+2 M \ln (r / 2 M-1)$ being the Regge-Wheeler tortoise coordinate. The physical interpretation of these modes is that $\vec{u}$ represents modes emerging from the past horizon and the $\overleftarrow{u}$ denotes those coming in from infinity. With the basics of the scalar field modes given above, we now apply the formalism outlined in the preceding section to calculate the Lamb shifts of the static atoms in three vacuum states of the quantum scalar fields respectively.

a. Boulware vacuum. The Boulware vacuum is defined by requiring normal modes to be positive frequency with respect to the Killing vector $\partial / \partial t$. One can show that the Wightman function for massless scalar fields in this vacuum state is given by [13, 14]

$$
D_{B}^{+}\left(x, x^{\prime}\right)=\frac{1}{4 \pi} \sum_{l m}\left|Y_{l m}(\theta, \varphi)\right|^{2} \int_{0}^{+\infty} \frac{d \omega}{\omega} e^{-i \omega \Delta t}\left[\left|\vec{R}_{l}(\omega \mid r)\right|^{2}+\left|\overleftarrow{R}_{l}(\omega \mid r)\right|^{2}\right]
$$


and the corresponding symmetric correlation function and linear susceptibility of the field are respectively

$$
\begin{gathered}
C^{F}\left(x(\tau), x\left(\tau^{\prime}\right)\right)=\frac{1}{8 \pi} \sum_{l m}\left|Y_{l m}(\theta, \varphi)\right|^{2} \int_{0}^{\infty} \frac{d \omega}{\omega}\left(e^{\frac{i \omega \Delta \tau}{\sqrt{g_{00}}}}+e^{-\frac{i \omega \Delta \tau}{\sqrt{g_{00}}}}\right) \times \\
{\left[\left|\vec{R}_{l}(\omega \mid r)\right|^{2}+\left|\overleftarrow{R}_{l}(\omega \mid r)\right|^{2}\right]} \\
\chi^{F}\left(x(\tau), x\left(\tau^{\prime}\right)\right)=\frac{1}{8 \pi} \sum_{l m}\left|Y_{l m}(\theta, \varphi)\right|^{2} \int_{0}^{\infty} \frac{d \omega}{\omega}\left(e^{-\frac{i \omega \Delta \tau}{\sqrt{g_{00}}}}-e^{\frac{i \omega \Delta \tau}{\sqrt{g_{00}}}}\right) \times \\
{\left[\left|\vec{R}_{l}(\omega \mid r)\right|^{2}+\left|\overleftarrow{R}_{l}(\omega \mid r)\right|^{2}\right]}
\end{gathered}
$$

where we have used $\Delta \tau=\sqrt{g_{00}} \Delta t$. Substituting the above statistical functions into Eqs. (11) and (12), extending the integration range for $\tau$ to infinity for sufficiently long times $\tau-\tau_{0}$, and performing the integration over $\tau$, we obtain the contribution of the vacuum fluctuations to energy level shift for an atom in state $|b\rangle$ held static at a distance $r$ from the black hole

$$
\begin{aligned}
\left(\delta E_{b}\right)_{v f}= & \frac{\mu^{2}}{32 \pi^{2} \hbar} \sum_{d}\left|\left\langle b\left|S_{2}(0)\right| d\right\rangle\right|^{2} \times \\
& \int_{0}^{\infty} d \omega\left(\frac{\omega}{\frac{\omega}{\sqrt{g_{00}}}+\omega_{b d}}-\frac{\omega}{\frac{\omega}{\sqrt{g_{00}}}-\omega_{b d}}\right)[\vec{g}(\omega \mid r)+\overleftarrow{g}(\omega \mid r)],
\end{aligned}
$$

and that of radiation reaction

$$
\begin{aligned}
& \left(\delta E_{b}\right)_{r r}=-\frac{\mu^{2}}{32 \pi^{2} \hbar} \sum_{d}\left|\left\langle b\left|S_{2}(0)\right| d\right\rangle\right|^{2} \times \\
& \quad \int_{0}^{\infty} d \omega\left(\frac{\omega}{\frac{\omega}{\sqrt{g 00}}+\omega_{b d}}+\frac{\omega}{\frac{\omega}{\sqrt{g 00}}-\omega_{b d}}\right)[\vec{g}(\omega \mid r)+\overleftarrow{g}(\omega \mid r)]
\end{aligned}
$$

where we have appealed to the following property of the spherical harmonics

$$
\sum_{m=-l}^{l}\left|Y_{l m}(\theta, \varphi)\right|^{2}=\frac{2 l+1}{4 \pi}
$$

and defined

$$
\begin{aligned}
& \vec{g}(\omega \mid r)=\frac{1}{\omega^{2}} \sum_{l=0}^{\infty}(2 l+1)\left|\vec{R}_{l}(\omega \mid r)\right|^{2} \\
& \overleftarrow{g}(\omega \mid r)=\frac{1}{\omega^{2}} \sum_{l=0}^{\infty}(2 l+1)\left|\overleftarrow{R}_{l}(\omega \mid r)\right|^{2}
\end{aligned}
$$

Since we do not have a generic expression for $R_{l}(\omega \mid r)$ functions, let us now examine in detail the energy level shifts both at close to the horizon and at infinity, which are anyway 
regions of physical interest. Then, the summations in Eqs. (24) and (25) can be simplified by the asymptotic properties of the radial functions [14]

$$
\begin{gathered}
\sum_{l=0}^{\infty}(2 l+1)\left|\vec{R}_{l}(\omega \mid r)\right|^{2} \sim \begin{cases}\frac{4 \omega^{2}}{1-\frac{2 M}{r}}, & r \rightarrow 2 M \\
\frac{1}{r^{2}} \sum_{l=0}^{\infty}(2 l+1)\left|B_{l}(\omega)\right|^{2}, & r \rightarrow \infty\end{cases} \\
\sum_{l=0}^{\infty}(2 l+1)\left|\overleftarrow{R}_{l}(\omega \mid r)\right|^{2} \sim \begin{cases}\frac{1}{4 M^{2}} \sum_{l=0}^{\infty}(2 l+1)\left|B_{l}(\omega)\right|^{2}, & r \rightarrow 2 M \\
\frac{4 \omega^{2}}{1-\frac{2 M}{r}}, & r \rightarrow \infty\end{cases}
\end{gathered}
$$

In Eq. (30), we have retained a factor $g_{00}^{-1}=(1-2 M / r)^{-1}$ for the asymptotic form at infinity which was omitted in Ref. [14] as it approaches to 1 when $r \rightarrow \infty$.

So, in these two asymptotic regions, we can write

$$
\begin{gathered}
\left(\delta E_{b}\right)_{v f}=\frac{\mu^{2}}{8 \pi^{2} \hbar} \sum_{d}\left|\left\langle b\left|S_{2}(0)\right| d\right\rangle\right|^{2} \int_{0}^{\infty} d \omega[1+f(\omega, r)] P\left(\frac{\omega}{\omega+\omega_{b d}}-\frac{\omega}{\omega-\omega_{b d}}\right) \\
\left(\delta E_{b}\right)_{r r}=-\frac{\mu^{2}}{8 \pi^{2} \hbar} \sum_{d}\left|\left\langle b\left|S_{2}(0)\right| d\right\rangle\right|^{2} \int_{0}^{\infty} d \omega[1+f(\omega, r)] P\left(\frac{\omega}{\omega+\omega_{b d}}+\frac{\omega}{\omega-\omega_{b d}}\right) .
\end{gathered}
$$

Here we have made a variable transformation, $\omega / \sqrt{g_{00}} \rightarrow \omega$, and defined

$$
f(\omega, r)=\frac{1}{4 r^{2} \omega^{2}} \sum_{l=0}^{\infty}(2 l+1)\left|B_{l}\left(\omega \sqrt{g_{00}}\right)\right|^{2},
$$

$P$ here and after denotes the principal value. Notice that for each definite initial state of a two-level atom, $|b\rangle, \sum_{d}\left|\left\langle b\left|S_{2}(0)\right| d\right\rangle\right|^{2}=1 / 4$, and the integrand in Eq. (32) is an even function of $\left|\omega_{b d}\right|=\omega_{0}$, so it is obvious that $\left(\delta E_{+}\right)_{r r}=\left(\delta E_{-}\right)_{r r}$. Radiation reaction has equal contribution to the energy shift of each level. Adding up the contributions of vacuum fluctuations and radiation reaction, we obtain the total energy level shift of the state $|b\rangle$,

$$
\delta E_{b}=-\frac{\mu^{2}}{4 \pi^{2} \hbar} \sum_{d}\left|\left\langle b\left|S_{2}(0)\right| d\right\rangle\right|^{2} \int_{0}^{\infty} d \omega[1+f(\omega, r)] P \frac{\omega}{\omega-\omega_{b d}} .
$$

So, the energy shift of the excited and ground states of the two-level atom are respectively

$$
\begin{aligned}
& \delta E_{+}=-\frac{\mu^{2}}{16 \pi^{2} \hbar} \int_{0}^{\infty} d \omega[1+f(\omega, r)] P \frac{\omega}{\omega-\omega_{0}} \\
& \delta E_{-}=-\frac{\mu^{2}}{16 \pi^{2} \hbar} \int_{0}^{\infty} d \omega[1+f(\omega, r)] P \frac{\omega}{\omega+\omega_{0}}
\end{aligned}
$$


The relative energy shift, i.e., the Lamb shift, which is an observable physical quantity, is then obtained by the subtraction, $\Delta=\delta E_{+}-\delta E_{-}$

$$
\begin{aligned}
\Delta_{B} & =\frac{\mu^{2}}{16 \pi^{2} \hbar} \int_{0}^{\infty} d \omega P\left(\frac{\omega}{\omega+\omega_{0}}-\frac{\omega}{\omega-\omega_{0}}\right) \\
& +\frac{\mu^{2}}{16 \pi^{2} \hbar} \int_{0}^{\infty} d \omega f(\omega, r) P\left(\frac{\omega}{\omega+\omega_{0}}-\frac{\omega}{\omega-\omega_{0}}\right) .
\end{aligned}
$$

Actually, the relative shift of the atomic energy is entirely caused by vacuum fluctuations and can be calculated directly by $\Delta=\left(\delta E_{+}\right)_{v f}-\left(\delta E_{-}\right)_{v f}$, because of the equal contribution of radiation reaction to the two levels. It is composed of two parts. The first part is just the Lamb shift of a two-level atom in a free Minkowski space-time with no boundaries. It is logarithmically divergent, which is expected for a nonrelativistic treatment as what we do here and the divergence can be removed by introducing a cutoff [15, 16] or resorting to a fully relativistic approach [17, 18]. The second part represents a finite correction to the Lamb shift in an unbounded flat space-time. It arises as a result of the back scattering of vacuum field modes off the space-time curvature of the black hole in much the same way as the reflection of the field modes at the reflecting boundary in a flat space-time, which also gives rise to corrections to the Lamb shift in the unbounded space [1, 4]. In fact, the second part of Eq. (37) gives the correction to the Lamb shift for an atom held static outside a massive spherical object with a radius larger than the Schwarzschild radius, since the Boulware vacuum is the natural vacuum outside such an object. This part can actually be further simplified. To do this, let us note that the coefficient $\left|B_{l}\right|$ can be approximated, using the geometrical optics approximation[12], by 1 for $l<\sqrt{27} M \omega$ and 0 for $l>\sqrt{27} M \omega$ so that $B_{l}(\omega) \sim \theta(\sqrt{27} M \omega-l)$. Thus, the summation in $f(\omega, r)$ can be evaluated to yield

$$
f(\omega, r) \approx \frac{27 M^{2} g_{00}}{4 r^{2}}=\frac{27 M^{2}}{4 r^{2}}\left(1-\frac{2 M}{r}\right) \equiv f(r),
$$

which does not depend on $\omega$. So, the second part is also logarithmically divergent and the divergence can again be dealt with as that in the first part. Denote the Lamb shift in a free Minkowski space-time by $\Delta_{M}$, we can write

$$
\Delta_{B}=[1+f(r)] \Delta_{M}
$$

Let us note that the correction vanishes both at infinity and at the even horizon, where the effective potential becomes zero, and it reaches the maximum value near $r=3 M$, where the effective potential has a peak and thus the vacuum field modes are most strongly scattered.

b. Unruh vacuum. Now we turn our attention to the case of the Unruh vacuum. The Wightman function for the real massless scalar field in this vacuum is given by [13, 14]

$$
\begin{aligned}
D_{U}^{+}\left(x, x^{\prime}\right)=\frac{1}{4 \pi} & \sum_{l m}\left|Y_{l m}(\theta, \varphi)\right|^{2} \int_{-\infty}^{+\infty} \frac{d \omega}{\omega} \times \\
& {\left[\frac{e^{-i \omega \Delta t}}{1-e^{-2 \pi \omega / \kappa}}\left|\vec{R}_{l}(\omega \mid r)\right|^{2}+\theta(\omega) e^{-i \omega \Delta t}\left|\overleftarrow{R}_{l}(\omega \mid r)\right|^{2}\right] }
\end{aligned}
$$


where $\kappa=1 / 4 M$ is the surface gravity of the black hole. The two statistical functions of the scalar field readily follow

$$
\begin{aligned}
& C^{F}\left(x(\tau), x\left(\tau^{\prime}\right)\right)=\frac{1}{8 \pi} \sum_{l m}\left|Y_{l m}(\theta, \varphi)\right|^{2} \int_{-\infty}^{\infty} \frac{d \omega}{\omega}\left(e^{\frac{i \omega \Delta \tau}{\sqrt{g_{00}}}}+e^{-\frac{i \omega \Delta \tau}{\sqrt{g_{00}}}}\right) \times \\
& {\left[\frac{\left|\vec{R}_{l}(\omega \mid r)\right|^{2}}{1-e^{-2 \pi \omega / \kappa}}+\theta(\omega)\left|\overleftarrow{R}_{l}(\omega \mid r)\right|^{2}\right] } \\
& \chi^{F}\left(x(\tau), x\left(\tau^{\prime}\right)\right)=\frac{1}{8 \pi} \sum_{l m}\left|Y_{l m}(\theta, \varphi)\right|^{2} \int_{-\infty}^{\infty} \frac{d \omega}{\omega}\left(e^{-\frac{i \omega \Delta \tau}{\sqrt{g_{00}}}}-e^{\frac{i \omega \Delta \tau}{\sqrt{g_{00}}}}\right) \times \\
& {\left[\frac{\left|\vec{R}_{l}(\omega \mid r)\right|^{2}}{1-e^{-2 \pi \omega / \kappa}}+\theta(\omega)\left|\overleftarrow{R}_{l}(\omega \mid r)\right|^{2}\right] }
\end{aligned}
$$

The contributions of vacuum fluctuations and radiation reaction can now be calculated by Eqs. (11) and (12) to yield

$$
\begin{aligned}
&\left(\delta E_{b}\right)_{v f}= \frac{\mu^{2}}{32 \pi^{2} \hbar} \sum_{d}\left|\left\langle b\left|S_{2}(0)\right| d\right\rangle\right|^{2} \int_{-\infty}^{\infty} d \omega \times \\
& {\left[\frac{1}{1-e^{-2 \pi \omega / \kappa}} \vec{g}(\omega \mid r)+\theta(\omega) \overleftarrow{g}(\omega \mid r)\right]\left(\frac{\omega}{\frac{\omega}{\sqrt{g_{00}}}+\omega_{b d}}-\frac{\omega}{\frac{\omega}{\sqrt{g_{00}}}-\omega_{b d}}\right), } \\
&\left(\delta E_{b}\right)_{r r}=-\frac{\mu^{2}}{32 \pi^{2} \hbar} \sum_{d}\left|\left\langle b\left|S_{2}(0)\right| d\right\rangle\right|^{2} \int_{-\infty}^{\infty} d \omega \times \\
& {\left[\frac{1}{1-e^{-2 \pi \omega / \kappa}} \vec{g}(\omega \mid r)+\theta(\omega) \overleftarrow{g}(\omega \mid r)\right]\left(\frac{\omega}{\frac{\omega}{\sqrt{g_{00}}}+\omega_{b d}}+\frac{\omega}{\frac{\omega}{\sqrt{g_{00}}}-\omega_{b d}}\right) . }
\end{aligned}
$$

Here again, one can see from Eq.(44) that $\left(\delta E_{+}\right)_{r r}=\left(\delta E_{-}\right)_{r r}$ for the same reasons as that in the Boulware vacuum. Similarly, we now focus our attention on the two asymptotic regions, i.e., when $r \rightarrow 2 M$ and $r \rightarrow \infty$. Adding up the contributions of vacuum fluctuations and radiation reaction, calculating out the energy shift for each level, and then performing a subtraction, we can derive the Lamb shift, which is, for an atom fixed near the event horizon, i.e., when $r \rightarrow 2 M$,

$$
\Delta_{U} \approx[1+f(r)] \Delta_{M}+\Delta_{T}
$$

where

$$
\Delta_{T}=\frac{\mu^{2}}{8 \pi^{2} \hbar} \int_{0}^{\infty} d \omega P\left(\frac{\omega}{\omega+\omega_{0}}-\frac{\omega}{\omega-\omega_{0}}\right) \frac{1}{e^{2 \pi \omega / \kappa_{r}}-1},
$$

and $\kappa_{r}=\kappa / \sqrt{g_{00}} . \Delta_{T}$, the correction term, as opposed to the Boulware vacuum case, is in the same form as the acceleration correction to the Lamb shift derived in [2] of a uniformly accelerated atom which would find itself in a thermal bath at the Unruh temperature, and is in structural similarity to the corresponding expressions obtained for the Lamb shift in a 
thermal heat bath [19 21] in that they have in common the appearance of the thermal factor $\left(e^{2 \pi \omega / \kappa_{r}}-1\right)^{-1}$. The difference can be attributed to the discrepancy between the scalar field we consider here and the electromagnetic field.

So, close to the horizon, the Lamb shift gets a correction, which is what one would find for a static atom if there is thermal radiation at the temperature

$$
T=\frac{\kappa_{r}}{2 \pi}=\frac{\kappa}{2 \pi \sqrt{g_{00}}}=\frac{T_{H}}{\sqrt{g_{00}}}
$$

with $T_{H}=\kappa / 2 \pi$, being the usual Hawking temperature of the black hole. Eq. (47) is the well-known Tolman relation [22, 23], which gives the proper temperature as measured by a local observer. Thus, a static atom close to the horizon of a black hole behaves, in terms of the Lamb shift, as if there is thermal radiation emanating from the black hole horizon. Notice that $T$ actually diverges as the horizon is approached, and this should come as no surprise since the atom must be in acceleration relative to the local free-falling frame to maintain at a fixed distance from the black hole, and this acceleration, which blows up at the horizon, gives rise to additional thermal effect. A comparison of Eq. (45) with Eq.(37) leads to the following relationship at the horizon

$$
\left.\Delta_{U}\right|_{r \rightarrow 2 M}=\left.\Delta_{B}\right|_{r \rightarrow 2 M}+\left.\Delta_{T}\right|_{r \rightarrow 2 M}
$$

For an atom fixed far from the black hole, i.e. when $r \rightarrow \infty$, the Lamb shift becomes

$$
\Delta_{U} \approx[1+f(r)] \Delta_{M}+f(r) \Delta_{T}
$$

Here we have used the relation $f(-\omega, r)=f(\omega, r)$ that can be deduced from the properties of $B_{l}(\omega)$ given in Ref. [12]. Different from that near the event horizon, the correction term, which is thermallike, is modified by a grey-body factor $f(\omega, r) \sim f(r)$. It can be understood as a result of backscattering of the outgoing thermal flux emanating from the event horizon off space-time curvature, which results in part of the outgoing flux being depleted. As the atom is placed further and further away, the thermal flux becomes weaker and weaker, and so is its contribution to the Lamb shift. Consequently,

$$
\begin{aligned}
\left.\Delta_{U}\right|_{r \rightarrow \infty} & \left.\approx \Delta_{B}\right|_{r \rightarrow \infty}+\left[f(r) \Delta_{T}\right]_{r \rightarrow \infty} \\
& \left.\approx \Delta_{B}\right|_{r \rightarrow \infty}=\Delta_{M} .
\end{aligned}
$$

c. Hartle-Hawking vacuum. Let us focus on the case of the Hartle-Hawking vacuum. The Wightman function for the real massless scalar field now is [13, 14]

$$
\begin{aligned}
& D_{H}^{+}\left(x, x^{\prime}\right)=\frac{1}{4 \pi} \sum_{l m}\left|Y_{l m}(\theta, \varphi)\right|^{2} \int_{-\infty}^{+\infty} \frac{d \omega}{\omega} \times \\
& {\left[\frac{e^{-i \omega \Delta t}}{1-e^{-2 \pi \omega / \kappa}}\left|\vec{R}_{l}(\omega \mid r)\right|^{2}+\frac{e^{i \omega \Delta t}}{e^{2 \pi \omega / \kappa}-1}\left|\overleftarrow{R}_{l}(\omega \mid r)\right|^{2}\right], }
\end{aligned}
$$


and it leads to the statistical functions of the scalar field as follows

$$
\begin{aligned}
C^{F}\left(x(\tau), x\left(\tau^{\prime}\right)\right)=\frac{1}{8 \pi} & \sum_{l m}\left|Y_{l m}(\theta, \varphi)\right|^{2} \int_{-\infty}^{+\infty} \frac{d \omega}{\omega}\left(e^{\frac{i \omega \Delta \tau}{\sqrt{g_{00}}}}+e^{\left.-\frac{i \omega \Delta \tau}{\sqrt{g_{00}}}\right)}\right. \\
& \times\left(\frac{\left|\vec{R}_{l}(\omega \mid r)\right|^{2}}{1-e^{-2 \pi \omega / \kappa}}+\frac{\left|\overleftarrow{R}_{l}(\omega \mid r)\right|^{2}}{e^{2 \pi \omega / \kappa}-1}\right) \\
\chi^{F}\left(x(\tau), x\left(\tau^{\prime}\right)\right)=\frac{1}{8 \pi} & \sum_{l m}\left|Y_{l m}(\theta, \varphi)\right|^{2} \int_{-\infty}^{+\infty} \frac{d \omega}{\omega}\left(e^{-\frac{i \omega \Delta \tau}{\sqrt{g_{00}}}}-e^{\frac{i \omega \Delta \tau}{\sqrt{g_{00}}}}\right) \\
& \times\left(\frac{\left|\vec{R}_{l}(\omega \mid r)\right|^{2}}{1-e^{-2 \pi \omega / \kappa}}-\frac{\left|\overleftarrow{R}_{l}(\omega \mid r)\right|^{2}}{e^{2 \pi \omega / \kappa}-1}\right)
\end{aligned}
$$

So, the contributions of vacuum fluctuations and radiation reaction to the energy shift of level $|b\rangle$ can be separately calculated to give

$$
\begin{aligned}
\left(\delta E_{b}\right)_{v f}= & \frac{\mu^{2}}{32 \pi^{2} \hbar} \sum_{d}\left|\left\langle b\left|S_{2}(0)\right| d\right\rangle\right|^{2} \int_{-\infty}^{\infty} d \omega \times \\
& \left(\frac{\omega}{\frac{\omega}{\sqrt{g_{00}}}+\omega_{b d}}-\frac{\omega}{\frac{\omega}{\sqrt{g_{00}}}-\omega_{b d}}\right)\left[\frac{\vec{g}(\omega \mid r)}{1-e^{-2 \pi \omega / \kappa}}+\frac{\overleftarrow{g}(\omega \mid r)}{e^{2 \pi \omega / \kappa}-1}\right] \\
\left(\delta E_{b}\right)_{r r}=-\frac{\mu^{2}}{32 \pi^{2} \hbar} \sum_{d}\left|\left\langle b\left|S_{2}(0)\right| d\right\rangle\right|^{2} \int_{-\infty}^{\infty} d \omega \times & \left(\frac{\omega}{\frac{\omega}{\sqrt{g_{00}}}+\omega_{b d}}+\frac{\omega}{\frac{\omega}{\sqrt{g_{00}}}-\omega_{b d}}\right)\left[\frac{\vec{g}(\omega \mid r)}{1-e^{-2 \pi \omega / \kappa}}-\frac{\overleftarrow{g}(\omega \mid r)}{e^{2 \pi \omega / \kappa}-1}\right]
\end{aligned}
$$

Using the above results, we can examine the behaviors of the Lamb shift in two asymptotic regions. First, when $r \rightarrow \infty$, we find

$$
\Delta_{H} \approx[1+f(r)] \Delta_{M}+f(r) \Delta_{T}+\Delta_{T}
$$

Here, the first correction term as opposed to the Boulware vacuum case is caused by the thermal radiation from the black hole which is backscattered by the space-time curvature and the backscattering is represented by the appearance of the grey-body factor, $f(\omega, r) \sim f(r)$, as we have already studied in the Unruh vacuum case. The second correction term $\left(\Delta_{T}\right)$ is the correction one would get for the Lamb shift in a thermal bath at the Hawking temperature $T_{H}$ (Here we have taken into account that $T=T_{H}$ at infinity.) Since the thermal radiation from the black hole diminishes to zero at infinity due to the backscattering off the curvature, as we have already pointed out, the presence of the thermal correction term, $\Delta_{T}$, indicates, in the Hartle-Hawking vacuum, that there is a thermal distribution of quanta at the Hawking temperature at infinity, and therefore, the Hartle-Hawking vacuum is not a vacuum in real sense, but a state that describes a black hole in equilibrium with an infinite sea of blackbody 
radiation. This is consistent with our understanding of the Hartle-Hawking vacuum gained in studies in other different contexts [10, 14, 24]. Note that Eq. (56) can also be written as

$$
\left.\Delta_{H}\right|_{r \rightarrow \infty}=\left.\Delta_{U}\right|_{r \rightarrow \infty}+\left.\Delta_{T}\right|_{r \rightarrow \infty} .
$$

Let us now study what happens at close to the horizon, i.e., when $r \rightarrow 2 M$. It then follows that

$$
\Delta_{H} \approx[1+f(r)] \Delta_{M}+[1+f(r)] \Delta_{T}
$$

Here the $\Delta_{T}$ term is a correction reminiscent of what one has for the Lamb shift close to the black hole horizon in the Unruh vacuum case, and it is a consequence of the outgoing thermal radiation from the black hole. The last term is a contribution of the incoming radiation of the thermal bath at infinity, which is backscattered off the curvature on its way to the black hole horizon. Eq. (58) can also be written as

$$
\left.\Delta_{H}\right|_{r \rightarrow 2 M}=\left.\Delta_{U}\right|_{r \rightarrow 2 M}+\left[f(r) \Delta_{T}\right]_{r \rightarrow 2 M} .
$$

It is interesting to note that the appearance of the $\Delta_{T}$ term in the Lamb shifts close to the horizon both in the Unruh and the Hartle-Hawking vacua (refer to Eq. (45) and Eq. (58)) supports the notion that the Hawking radiation of a black hole originates from the black hole horizon, since $\Delta_{T}$ represents a contribution of purely thermal radiation not scattered by the space-time curvature.

Finally, it is worth pointing out that the correction term $\Delta_{T}$, which appears in the Lamb shifts in both the Unruh and Hartle-Hawking vacuua, is finite, and this can be seen by a careful inspection of the integral involved. Here, we would like to go a little bit further. We will analyze the behaviors of $\Delta_{T}$ both in the high and low temperature limits. For this purpose, we rewrite it as

$$
\Delta_{T}=-\frac{\mu^{2} \omega_{0}}{8 \pi^{2} \hbar} \int_{0}^{\infty} d x P\left(\frac{1}{x+x_{0}}+\frac{1}{x-x_{0}}\right) \frac{1}{e^{x}-1}
$$

with $x=\omega / T$ and $x_{0}=\omega_{0} / T$. When the Hawking temperature is low, that is, when $x_{0} \gg 1$, the integral can be approximated by

$$
\Delta_{T}=\frac{\mu^{2}}{8 \hbar}\left[\frac{T^{2}}{3 \omega_{0}}+\frac{2 \pi^{2} T^{4}}{15 \omega_{0}^{3}}+O\left(\frac{1}{x_{0}^{6}}\right)\right] .
$$

While in the high temperature limit, i.e., when $x_{0} \ll 1$, we have

$$
\Delta_{T} \approx \frac{\mu^{2} \omega_{0}}{8 \pi^{2} \hbar} \frac{1}{x_{0}}\left[e^{-x_{0}} \overline{E i}\left(x_{0}\right)-e^{x_{0}} \overline{E i}\left(-x_{0}\right)\right],
$$

where $\operatorname{Ei}(z)=-\int_{-z}^{\infty} \frac{e^{-t}}{t} d t$ and the overline denotes the principal value. Further simplifications yield

$$
\Delta_{T} \approx \frac{\mu^{2} \omega_{0}}{4 \pi^{2} \hbar}\left[1-\gamma+\ln \left(T / \omega_{0}\right)\right]+O\left(x_{0}^{2}\right)
$$

with $\gamma=0.577216$ being the Euler's constant. So, in the high temperature regime, the Lamb shift increases logarithmically with the temperature. 


\section{SUMMARY}

Using the formalism suggested by Dalibard, Dupont-Roc and Cohen-Tannoudji(DDC) [8, 9] which allows a distinct separation of the contributions of vacuum fluctuations and radiation reaction, we have calculated the Lamb shift of a static two-level atom interacting with real massless scalar fields in the Boulware, Unruh and Hartle-Hawking vacuums outside a Schwarzschild black hole.

In the Boulware vacuum case, we find that the Lamb shift gets a correction, as opposed to that in an unbounded flat space, which is reminiscent of the correction to the Lamb shift induced by the presence of boundaries, and it can be understood as a result of the back scattering of vacuum field modes off the space-time curvature of the black hole in much the same way as the reflection of the field modes at the reflecting boundary in a flat space-time. This correction can be viewed as the correction to the Lamb shift for an atom held static outside a massive spherical object with a radius larger than the Schwarzschild radius, and it reduces, at infinity, to the Lamb shift in a unbounded flat space as expected.

In the Unruh vacuum case, we find that the Lamb shift is corrected by a thermal term, as compared to that in Boulware vacuum case, which can be regarded as a result of the thermal radiation at the Hawking temperature emanating from the black hole horizon and the additional thermal effect due to the acceleration relative to the local free-falling frame the atom must have in order to be static. However, the thermal radiation from the black hole is backscattered off the space-time curvature, and becomes weaker and weaker on its way away from the black hole, rendering the Lamb shift to reduce to that in a flat space at infinity.

However, when the Hartle-Hawking vacuum is concerned, our results show that the correction to the Lamb shift close to the horizon, as opposed to that in the Boulware vacuum, would be a purely thermal contribution term due to an outgoing Hawking radiation from black hole plus that due to an incoming radiation from infinity which is backscattered off the curvature, whereas, at infinity, the Lamb shift gets corrected by a purely thermal term characteristic of a sea of black-body radiation plus a term resulting from the backscattered Hawking radiation from the black hole. This supports the notion that the Hartle-Hawking vacuum is a state that describes a black hole in equilibrium with an infinite sea of blackbody radiation, rather than a vacuum state in the usual sense.

\section{Acknowledgments}

One of us (WZ) would like to thank Jialin Zhang for helpful discussions. This work was supported in part by the National Natural Science Foundation of China under Grants No. 10775050, No. 11075083, and No. 10935013; the Zhejiang Provincial Natural Science Foundation of China under Grant No. Z6100077; the SRFDP under Grant No. 20070542002; the 
National Basic Research Program of China under Grant No. 2010CB832803; the PCSIRT under Grant No. IRT0964; and the Programme for the Key Discipline in Hunan Province.

[1] D. Meschede, W. Jhe and E.A. Hinds, Phys. Rev. A 41, 1587 (1990).

[2] J. Audretsch and R. Müller, Phys. Rev. A 52, 629 (1995).

[3] R. Passante, Phys. Rev. A 57, 1590 (1998).

[4] L. Rizzuto, Phys. Rev. A 76, 062114 (2007).

[5] Z. Zhu and H. Yu, Phys. Rev. A, (to be published).

[6] W. G. Unruh, Phys. Rev. D 14, 870 (1976).

[7] J. Hartle and S. Hawking, Phys. Rev. D13, 2188 (1976).

[8] J. Dalibard, J. Dupont-Roc and C. Cohen-Tannoudji, J. Phys. (France)43, 1617 (1982).

[9] J. Dalibard, J. Dupont-Roc and C. Cohen-Tannoudji, J. Phys. (France)45, 637 (1984).

[10] H. Yu and W. Zhou, Phys. Rev. D, 76, 027503 (2007); ibid, 76, 044023 (2007).

[11] J. Audretsch and R. Müller, Phys. Rev. A 50, 1755 (1994).

[12] B. S. DeWitt, Phys. Rep. 19, 295 (1975).

[13] S. M. Christensen and S. A. Fulling, Phys. Rev. D 15, 2088 (1977).

[14] P. Candelas, Phys. Rev. D 21, 2185 (1980).

[15] H. A. Bethe, Phys. Rev. 72, 339 (1947).

[16] T. A. Welton, Phys. Rev. 74, 1157 (1948).

[17] N. M. Kroll and W. E. Lamb, Phys. Rev. 75, 388 (1949).

[18] J. B. French and V. F. Weisskopf, Phys. Rev. 75, 1240 (1949).

[19] G. Barton, Phys. Rev. A 5, 468 (1972).

[20] P. L. Knight, J. Phys. A 5, 417 (1972).

[21] J. W. Farley and W. H. Wing, Phys. Rev. A 23, 2397 (1981).

[22] R. Tolman, Phys. Rev. 35, 904 (1930).

[23] R. Tolman and P. Ehrenfest, Phys. Rev. 36, 1791 (1930).

[24] J. Zhang and H. Yu, Phys. Rev. D 75, 104014 (2007). 